\title{
Emulgel: A Boon for Enhanced Topical Drug Delivery
}

\author{
Narayana Rompicherla Charyulu, Punam Joshi, Akhilesh Dubey, Amitha Shetty* \\ Department of Pharmaceutics, NGSM Institute of Pharmaceutical Sciences, NITTE (Deemed to be University), Mangaluru, Karnataka, INDIA.
}

\begin{abstract}
Background: Topical drug delivery system is dosage forms which are applied directly to the skin to cure various diseases. When we compare all the semisolid preparation for topical drug delivery, gels have been extensively used in cosmetics as well as pharmaceutical preparations. The major drawback of gels is in delivery of hydrophobic drugs which can be overcome by using emulgels. The dosage forms where gel and emulsion are combined together are known as emulgel. Emulgel is one of the modern technologies in novel drug delivery system for topical use having properties of dual control release i.e. emulsion and gel. Methods: In emulgel drug delivery system drugs are incorporated into globules which are then dispersed resulting in the formation of emulsion which is then mixed with the gel base. Results: It was seen that emulgels are better topical drug delivery systems compared to others owing to its advantages such as greaseless, smooth, homogeneous texture, glossy appearance, transparent, $\mathrm{pH}$ equivalent to $\mathrm{pH}$ of skin, high drug content and sustained
\end{abstract}

release. Conclusion: This review article focuses on emulgel including its properties and formulation considerations with characterization parameters proving that emulgels are one of the most effective and convenient drug delivery systems.

Key words: Dual control release, Emulgel, Novel drug delivery system, Thixotropic, Topical drug delivery system.

\section{Correspondence}

Dr. Amitha Shetty

Assistant Professor, Department of Pharmaceutics, NGSM Institute of Pharmaceutical Sciences, NITTE (Deemed to be University), Deralakatte, Mangaluru-575018, Karnataka, INDIA.

Phone: +919449593585

Email: amithashetty@nitte.edu.in

DOI: 10.5530/jyp.2021.13.17

\section{INTRODUCTION}

Drugs are administered to human body through different routes such as oral, parenteral, rectal, sublingual, etc. ${ }^{1}$ Oral routes are considered to be the versatile route, however still it has disadvantages such as poor solubility and bioavailability. In such scenario topical drug delivery system can be considered as an option. The topical system bypasses the first pass metabolism, problems related to intravenous therapy, avoidance of the risks related to absorption, such as gastric emptying time, various enzymes, $\mathrm{pH}$ changes. But the main disadvantage of topical drug delivery system as a gel is hydrophobic moiety cannot be delivered through skin easily. ${ }^{2}$

Emulgels are basically emulsions, which are w/o or o/w type that are gelled in which gelling agent is used for mixing. Emulgels are best vehicle for hydrophobic drugs and are stable. Patient acceptability is high in case of emulgels as they have the advantages of both gels and emulsions. As emulgels possess the properties of both emulsion and gel, thus it acts as dual controlled release systems as shown in Figure 1. Emulgels has an ability to deliver lipophilic as well as hydrophilic drugs as they contain two phases, aqueous and non-aqueous. Emulgels are applied to skin suitably as it is non-greasy when compared to other topical preparations that requires excess rubbing.,

\section{MATERIALS}

The requirements and properties of chemical moieties of an emulgel is depicted in Table $1 .{ }^{5}$

\section{Drug}

The physicochemical and biological properties of drugs for topical or transdermal delivery is applicable for selecting the drug for emulgel formulation. ${ }^{6}$

\section{Vehicle}

The vehicle used for formulation of emulgels must possess properties such as should effectively deposit and evenly distribute the drug, release the drug for free migration of drug to the site of action, effectively deliver the drug to the appropriate target sites and sustain the therapeutic level of drug for a longer duration. ${ }^{6}$

\section{Aqueous Materials}

The aqueous phase of the emulsion is formed by using aqueous materials. Alcohol and water are commonly used to prepare aqueous phase. ${ }^{6}$

\section{Oil}

Castor oils and mineral oils that are non-biodegradable are utilized for local laxative effect. Oils those are extracted from various types of plants having different medicinal values shall be used in preparation of emulgels. ${ }^{6}$

\section{Emulsifier}

The emulsifying agents are used to boost emulsification during manufacturing as well as to maintain the stability during the shelf life e.g. sorbitan mono-oleate, polyethylene glycol 40 stearate, sodium stearate and stearic acid. ${ }^{6}$

\section{Gelling agents}

The consistency and the gelling property can be increased by using various gelling agents. An inverse relationship between the concentration of the gelling agent and the drug release can be observed. Carbopol and HPMC is often used in emulgel. ${ }^{6}$ 


\section{Penetration Enhancers}

Penetration enhancer's increases the drug permeability through the skin. Absorption of the drug through the skin can be promoted by using vehicles like penetration enhancers which for a short period disturbs the extremely ordered structure of the fluidize lipid channels of the stratum corneum that enhances the drug delivery into skin and partitioning of the drug into the skin. ${ }^{6}$

\section{Humectant}

Humectants are used to prevent loss of moisture from the formulation. They minimize drying of emulgels and thereby enhance qualities such ease of application and consistency. Examples of humectants include glycerine, propylene glycol etc. ${ }^{6}$

\section{METHOD}

\section{Emulgel preparation}

The preparation of emulgel includes three steps; first step is to prepare $\mathrm{O} / \mathrm{W}$ or W/O type emulsions in which the drug is included, then in second step the gel base is formulated and finally in the third step emulsion is added to the gel base by continuous stirring to produce an emulgel (Figure 2).

\section{Characterization of emulgels}

\section{Physical examination}

The emulgel shall be visually inspected for colour, consistency, homogeneity and phase separation. ${ }^{8}$

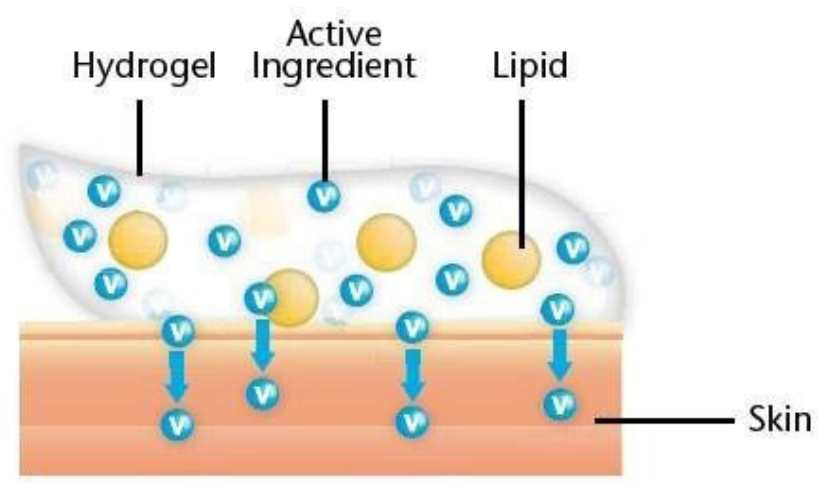

Figure 1: Structure of Emulgel.

Table 1: Primary requirements of chemical moiety.

\begin{tabular}{cc}
\hline Properties & Criteria \\
\hline Effective concentration & less than $10 \mathrm{mg}$ \\
$\mathrm{t}_{1 / 2}$ & $\leq 10 \mathrm{hr}$. \\
Molecular mass & 800 Dalton or less; desirably 500 Dalton or less \\
$\log p$ value & 0.8 to 5 \\
Skin permeability coefficient & $0.5 \times 10-3 \mathrm{~cm} / \mathrm{hr}$. \\
Irritation to skin & Non-irritating \\
Polarity & Less \\
Molecular size & Small \\
\hline
\end{tabular}

\section{pHDetermination}

The digital $\mathrm{pH}$ meter is used to measure the $\mathrm{pH}^{8}$

\section{Size of globule and its distribution analysis}

The size of the globules and its distribution is calculated using Malvern zeta sizer instrument. The mean globule diameter and distribution is determined. ${ }^{8}$

\section{Drug content}

The drug content of the emulgel is determined by dissolving a known quantity of emulgel in solvent, suitable dilutions are made and the solution is filtered. The absorbance is measured using the UVspectrophotometer and the drug content is determined. ${ }^{9}$

\section{In-vitro drug release}

The in-vitro drug release studies of emulgel can be carried out using modified diffusion cell having a dialysis membrane. The emulgel is spread uniformly on the dialysis membrane. The phosphate buffer taken in beaker is used as the receptor compartment. The donor compartment is kept in contact with the receptor compartment. The solution is continuously stirred. The samples are then spectrophotometrically analysed and the cumulative percentage drug release is determined. ${ }^{10}$

\section{In vitro drug release kinetic study}

The in vitro release kinetic study is utilised to examine the mechanism of drug release, then the data of drug release can be fitted into below equations:

Zero order equation: $\mathrm{Q}=\mathrm{k} 0 \mathrm{t}$

First order equation: In $(100-\mathrm{Q})=\mathrm{In} 100-\mathrm{k} 1 \mathrm{t}$

Higuchi's equation: $\mathrm{Q}=\mathrm{k} 2 \mathrm{~V}_{\mathrm{t}}$

Where, $\mathrm{Q}$ is percentage of drug release at time interval $\mathrm{t}, \mathrm{k} 0$ is zero order rate constant, $\mathrm{k} 1$ is first order rate constant and $\mathrm{k} 2$ is drug diffusion rate constant. ${ }^{10}$

\section{Skin Irritation Study (Patch Test)}

The skin irritation study of emulgels can be determined by using shaven skin of rat or rabbit. The weighed sample of emulgel is applied to either site to the area of the skin and keep them in cage for next $24 \mathrm{hr}$. After 24 hr examine the rat or rabbit skin area and check for any colour change or any adverse effect noted. If no adverse effect were found formulation passes the test. ${ }^{10}$

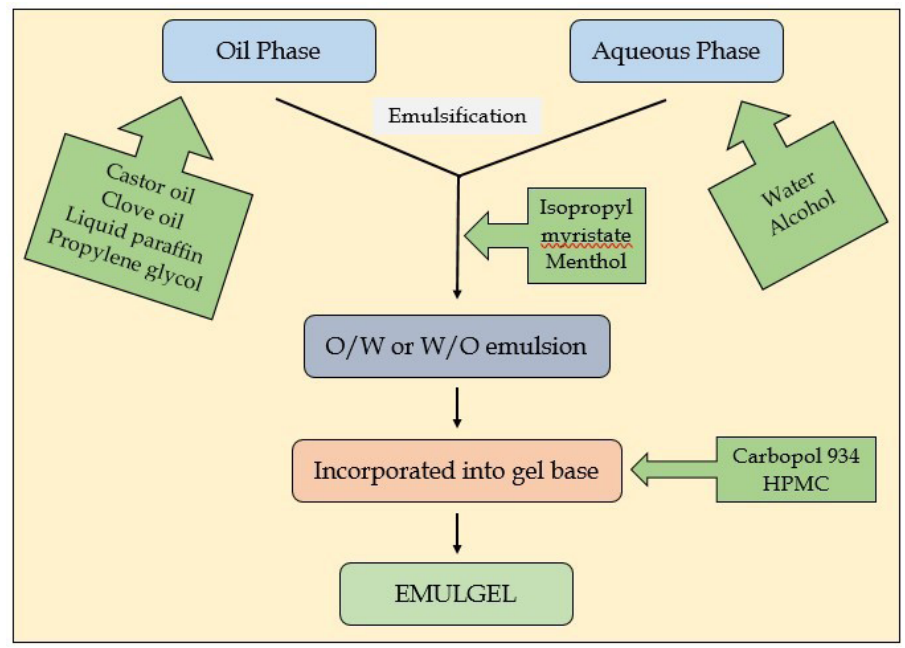

Figure 2: Method of formulation of Emulgel. 


\section{Ex vivo skin permeation studies}

The ex vivo skin permeation study can be conducted in the male rats. The full thick abdominal skin is removed and kept for hydration for $1 \mathrm{hr}$ and then mounted on to Franz diffusion cell. The emulgel is applied on skin and fixed onto the Franz diffusion cell. The receptor compartment contains the medium and stirred continuously. The aliquots are withdrawn and estimated spectrophotometrically. ${ }^{11}$

\section{RESULTS}

\section{Physical examination}

Emulgels will be white or transparent to white opaque and smooth homogeneous appearance with no significant phase separation.

\section{pHDetermination}

The $\mathrm{pH}$ of the emulgel formulations should be in the range of 6 to 6.5 , which lies in the normal $\mathrm{pH}$ range of the skin.

\section{Size of globule}

The size of the globules are measured in $\mu \mathrm{m}$ and should be as small as possible.

\section{Drug content}

Drug content values shall range from $95 \%$ to $98 \%$.

\section{In vitro drug release}

Emulgel formulations shows good drug release of more than $98 \%$ at the end of 5 hours.

\section{In vitro drug release kinetic study}

Emulgel formulations from the kinetic data usually shows drug release by zero order kinetics and higuchi mechanism i.e., diffusion. The ' $n$ ' values of korsmeyer- Peppas model indicates super case II mechanism.

\section{Skin Irritation Study (Patch Test)}

Emulgel formulations should show no redness or swelling during the 24 hr test period.

\section{Ex vivo skin permeation studies}

Emulgel formulations with penetration enhancers shows enhanced drug diffusion compared to the formulations without penetration enhancers.

\section{DISCUSSION}

The transdermal route is an alternative route of drug delivery for systemic effect and emulgel formulation is considered ideal for transdermal delivery. The formulation procedure is simple, inexpensive and less time consuming. ${ }^{12}$ Emulgel formulations were white viscous creamy preparation with a smooth homogeneous texture and glossy appearance add on as an advantage for patient acceptance. The $\mathrm{pH}$ of the emulgel formulation are in range as that of skin $\mathrm{pH}$ thereby avoids the risk of skin irritation upon application to skin. Amount of drug in the emulgel indicates the suitability of the system for high entrapment in the internal phase. Emulgel formulations show sustain release for a period of about 6 hr. The ' $n$ ' values of korsmeyer- Peppas model indicates that drug release is by erosion of polymeric chain. ${ }^{13}$

The marketed preparation of emulgels are depicted in Table $2 .{ }^{12}$ Yadav $\mathrm{S}$ et al. successfully formulated Tolnaftate emulgel with eucalyptus oil as penetration enhancers. Emulgel formulation with 5\% eucalyptus oil revealed improves in vitro drug release and ex vivo diffusion. ${ }^{14}$ Pakhare $\mathrm{AV}$ et al. formulated emulgel preparation containing diclofenac sodium showed excellent gelling and dispersion properties. They concluded that emulgel preparation is the only choice for water insoluble drugs with enhanced drug release. ${ }^{15}$
Table 2: List of marketed formulations.

\begin{tabular}{ccc}
\hline Brand name & Active ingredient & Manufacturer \\
\hline Voltarol Emulgel & $\begin{array}{c}\text { Diclofenac } \\
\text { Diethylammonium }\end{array}$ & Novartis \\
Diclomax Emulgel & Diclofenac sodium & Torrent Pharma \\
Miconaz-H- & Miconazole nitrate, & Medical union \\
Emulgel & Hydrocortisone & Pharmaceuticals \\
Dermafeet Emulgel & Urea & Herbitas \\
Isofen Emulgel & Ibuprofen & Beit jala pharmaceutical \\
Diclona Emulgel & Diclofenac diethylamine & Kuwait Saudi \\
Dosanac emulsion & Diclofenac & pharmaceutical \\
gel & diethylammonium & Siam bheasach \\
Diclon Emulgel & Diclofenac diethylamine & Med pharma \\
Cataflam Emulgel & Diclofenac potassium & Novartis \\
Denacine Emulgel & Clindamycin phosphate & Beit jala pharmaceutical \\
Avindo Gel & Azithromycin & Cosme Pharmaceuticals \\
\hline
\end{tabular}

\section{CONCLUSION}

After a thorough literature survey, we can conclude that emulgels has proven to be one of the most effective, better and convenient drug delivery systems as emulgels has the ability to deliver hydrophobic as well as hydrophilic drugs. As emulgel is non-greasy and has gel like property in which the gel serves as an aqueous environment to the drug which helps in dissolution therefore emulgels yields better drug release when compared to the other transdermal drug delivery systems. Since emulgel shows enhanced adhesion, spread ability, extrusion and viscosity, thus emulgel can be considered as an emerging novel drug delivery system with enhanced characteristics and advantages.

\section{REFERENCES}

1. Thomas J, Kuppuswamy S, Sahib A, Benedict A, George E. A review on emulge as a current trend in topical drug delivery system. Int J Pharm Pharm Res. 2017;9(3):273-81.

2. Yadav S, Mishra M, Tiwari A, Shukla A. Emulgel: A new approach for enhanced topical drug delivery. Int J Curr Pharm Res. 2017;9(1):15-9.

3. Begum S, Chetty M, Voleti V, Pavithra B, Akhila B, Gayathri C, et al. A review on emulgels: A novel approach for topical drug delivery. Asian Journal of Pharmaceutical Research and Development. 2019;7(2):70-7.

4. Sharma S. Topical preparations are used for the localized effects at the site of their application by virtue of drug penetration into the underlying layers of skin or mucous membranes. Pharmaceutical Reviews. 2008;6(1):26-39.

5. Ashara K, Chavda J, Soniwala M, Mendapara V, Mori N. To study effect of polymer and its proportions on release profile of erosion based tablet. Mintage J Pharm Med Sci. 2013;2:63-6.

6. Yassin G. Formulation and evaluation of optimized clotrimazole emulge formulations. Br J Pharm Res. 2014;4(9):1014-30.

7. Haneefa K, Mohanta G, Nayar C. Emulgel: An advanced review. J Pharm Sci Res. 2013;5(12):254-8.

8. Sah S, Badola A, Nayak B. Emulgel: Magnifying the application of topical drug delivery. Indian J Pharm Biol Res. 2017;5(1):25-33.

9. Joshi B, Singh G, Rana A, Saini S. Development and characterization of clarithromycin emulgel for topical delivery. Int J Drug Deliv Res. 2012;4(3):310-3.

10. Upadhyaya S, Chauhan B, Kothiyal P. Emulgel: A boon for dermatological diseases. Int J Pharm Res All Sci. 2014;3(4):1-9.

11. Thanushree $H$, Kiran G, Acharya A. Formulation development of diclofenac sodium emulgel using aloe vera gel for transdermal drug delivery system. Int J Pharm Sci Nanotech. 2017;10(5):3858-65.

12. Shivappa N, Arjun M, Rohini B, Ganesh N. Design, development and evaluation of microemulgel containing econazole nitrate. Int J Curr Res. 2018;10(8):72727 33 
13. Kapoor D, Vyas R, Lad C, Patel M, Lal B, Parmar R. Formulation, development and characterization of emulgel of a NSAID'S. Pharm Chem J. 2014;1(3):9-16.

14. Yadav S, Wairkar S, Invally M, Ranade S. Topical emulgel of tolnaftate with penetration enhancer: Development, characterisation and antifungal activity.
Indian J Med Res Pharm. 2017;4(10):28-35.

15. Pakhare AV, Deshmane SV, Deshmane SS, Biyani KR. Design and development of emulgel preparation containing diclofenac potassium. Asian $\mathrm{J}$ Pharm. 2017;11(4):SS712-6.

Article History: Submission Date : 15-10-2020; Revised Date : 09-01-2021; Acceptance Date : 25-01-2021

Cite this article: Charyulu NR, Joshi P, Dubey A, Shetty A. Emulgel: A Boon for Enhanced Topical Drug Delivery. JYoung Pharm. 2021;13(1):76-9. 